\title{
Influence of impurities on ion temperature measurements in the tokamak scrape-off layer by retarding field analyzers
}

\author{
M. Kočan and J. P. Gunn ${ }^{1}$ \\ Max-Planck-Institut für Plasmaphysik, Bolzmannstr. 2, D-85748 Garching, Germany. \\ ${ }^{1}$ CEA, IRFM, F-13108 Saint-Paul-lez-Durance, France. \\ E-mail: martin.kocan@ipp.mpg.de
}

\begin{abstract}
Retarding field analyzers (RFA) are one of the only widely accepted diagnostics for measuring ion temperature $T_{i}$ in the tokamak plasma boundary. Usually a pure, Maxwellian plasma composed of a single fuel ion species of charge $Z$ is assumed, in which case the RFA would measure $T_{i} / Z$. Incident impurity ion fluxes are neglected. We study the influence of impurity ions on $T_{i}$ inferred from a RFA by simulating measurements in a plasma with several ion species. We show that for typical impurity ion fluxes in the tokamak plasma boundary and in the collisionless limit of the kinetic simulations, the assumption of plasma purity causes $T_{i}$ to be underestimated by as much as $\sim 20 \%$.
\end{abstract}

PACS: 52.25.Vy, 52.25.Xz, 52.55.Fa, 52.65.-y, 52.65.Ff, 52.70.Ds

\section{Introduction}

Ion temperature $T_{i}$ is among the most important parameters of the tokamak scrape-off layer (SOL). Most often SOL $T_{i}$ is measured by retarding field analyzers (RFA) (see references in [1]). Other diagnostic methods for SOL $T_{i}$ measurements are used only sporadically ([1] and references therein) and rarely simultaneously with RFAs [2-4], so that experimentalists have to rely upon data from a single technique. Therefore, significant effort has been devoted to validate the assumptions of the RFA technique. Previous studies focusing on a number of potential instrumental effects concluded that for most operating conditions RFA $T_{i}$ measurements can be trusted, provided that the analyzer fulfills basic design requirements [3-12]. This paper contributes to these studies by investigating the effect of impurity ions on RFA $T_{i}$ measurements.

RFAs do not directly measure $T_{i}$, but $T_{i} / Z$, meaning that a charge state of the incident ions $Z$ has to be assigned. The absolute flux of each impurity charge state is rarely known in general $[10,11,13-15]$. Nonetheless, the impurity ions are considered as a small, unperturbing, minority by assuming $Z=Z_{\text {fuel }}$, the charge of fuel ions (typically $Z=1$ for deuterium $\mathrm{D}^{+}$). Impurity ions can increase the effective $Z$ and lead to an underestimation of $T_{i}$ inferred from a RFA.

The effect of impurity ions on RFA $T_{i}$ measurements in the DITE tokamak was estimated by taking into account the impurity ion fluxes monitored by a plasma ion mass 
spectrometer (PIMS) [9, 13]. In hydrogen plasmas, the assumption $Z=Z_{\text {fuel }}$ resulted in an underestimation of $T_{i}$ by almost a factor of 3 [9]. The findings in Ref. [9] are likely extreme in the sense that conditions in the DITE tokamak did not match the cleanliness of the majority of today's devices. Coupled with its nature as a limiter machines, SOL impurity fluxes in DITE were certainly elevated in comparison with more recent divertor tokamaks [16]. Nevertheless, the observations in Ref. [9] do suggest that even somewhat lower impurity concentrations in the flux sampled by the RFA can affect the measured ion temperature and the assumption $Z=Z_{\text {fuel }}$ has to be regarded cautiously. In the Tore Supra tokamak, SOL $T_{i}$ measured by a RFA was a factor of 4 lower than that obtained from spectroscopic measurements [4]. This disagreement can be associated with a number of other factors [4], yet it casts further doubts on the assumption $Z=Z_{\text {fuel }}$ and raises the following questions: How do impurity ions, even in low concentrations, generally influence the interpretation of RFA $T_{i}$ measurements? Also, can the effect of impurity ions on $T_{i}$ measurements be reduced using appropriate data analysis?

In this paper, we simulate the measurements of a RFA exposed to an impure plasma. To make the model as realistic as possible we account for the influence of the pre-sheath in front of the RFA, the acceleration of ions in the Debye sheath and the ion transmission through the RFA slit [12], neglected in an earlier study [9]. We show that for typical impurity concentrations in the SOL, the impurity ions have little effect on RFA measurements, provided that $T_{i}$ is inferred from the high-energy part of the ion current-voltage (I-V) characteristic.

Section 2 describes how RFAs work in pure plasmas. A brief survey of typical impurity ion fluxes in the tokamak SOL is given in section 3. Results of simulations of RFA measurements in a plasma with different impurity mixtures are described in section 4 . Conclusions are given in section 5 .

\section{RFA principle}

There are numerous variants of RFA construction. Here we treat the type of analyzer that is frequently used in the highly magnetized, tokamak SOL. It consists of a set of semipermeable grids and a collector, separated from the plasma by a thin plate in which a narrow slit is cut (Fig. 1). The slit plate serves to protect the delicate grids from intense plasma irradiation, while admitting enough ions to produce a measurable current on the collector. The slit plate is perpendicular to the magnetic field direction so that the RFA is sensitive to the parallel velocity of incident ions. The slit plate is biased negatively $\left(V_{s p}\right)$ to repel most incident electrons back into the plasma. A fraction of the incident ions are transmitted through the slit into the analyzer where they encounter the first grid, labelled as "grid 1", to which a positive voltage $V_{g 1}$ is applied. The ions that have enough kinetic energy to overcome $V_{g 1}$ then proceed to a second grid, labelled as "grid 2", biased to high negative voltage, and placed between grid 1 and the collector. Grid 2 is used to repel any remaining incident electrons that are energetic enough to penetrate $V_{s p}$, as well as to suppress secondary electron fluxes emitted inside the analyzer by ion impact. The ion current to the collector $I_{c}$ is a function of $V_{g 1}$,

$$
I_{c}=A_{s l i t} e \xi_{g}^{n} \sum_{j} Z_{j} \int_{u_{j}}^{\infty} d v_{j} v_{j} \xi_{r}\left(v_{j}\right) f_{j}\left(v_{j}\right)
$$


where the summation is over each charge of each ion species collected by the RFA. $A_{\text {slit }}$ is the slit area. $\xi_{g}$ is the grid transmission factor, with $n$ being the total number of grids between the slit plate and the collector ( $n$ depends on particular RFA design). $\xi_{r}$ is the relative slit transmission factor which is independent of the ion species [12]. In Eq. (1), $f_{j}$ are the parallel ion velocity distributions at the slit entrance. $u_{j}^{2}=2 e Z_{j} V_{g l} / m_{j}$ (with $m_{j}$ being the ion masses) is the minimum ion velocity at the slit plate needed to overcome the repelling force of grid 1. $I_{c}$ is maximum for $V_{g 1}=0$ (all ions reach the collector). Above a threshold voltage $V_{s}$ equal to the voltage drop across the collisionless Debye sheath in front of the slit plate, $I_{c}$ decreases monotonically to zero with increasing voltage.

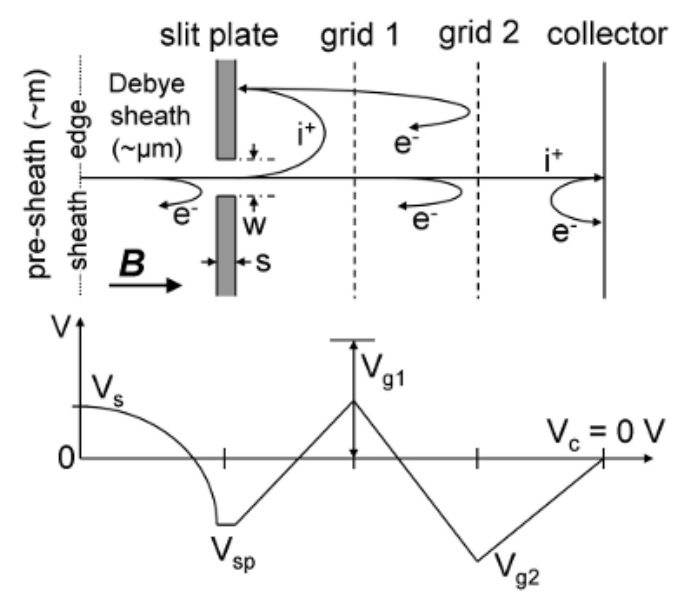

Figure 1. Schematic of main components of the RFA (not to scale) and the bias voltages typically applied for $T_{i}$ measurements.

It is standard practice to assume that the contribution of impurity fluxes to the collector current is negligible. In theory then, according to the single-species form of Eq. (1), the ion current characteristic could be differentiated to obtain the parallel speed distribution directly. Unfortunately this is not feasible because SOL turbulence causes the collector current to fluctuate faster than the grid voltage can be swept. Instead, the 1D collisionless model of Chung and Hutchinson [17] is used to predict the form of the ion current characteristic for the expected range of SOL plasma parameters, and empirical fits to the numerical results provide a means to interpret the smoothed experimental data. In accordance with measurements of near sonic ion flows, the fuel ions in the unperturbed SOL are assumed to have a drifting Maxwellian distribution of parallel speeds. For relevant SOL parameters (i.e. ion-to-electron temperature ratio $\tau=T_{i} / T_{e}>1$ ), it is found that the collector current is well approximated by a decaying exponential in the high voltage range. The e-folding voltage deduced from the data is equated to an effective ion temperature

$$
\frac{T_{i^{*}}}{Z}=\left(\frac{d \ln I_{c}}{d V_{g 1}}\right)^{-1}
$$

The flow introduces an asymmetry in $f$ which results in different effective $T_{i^{*}}$ on each side of the probe, $T_{i^{*}}^{u p}>T_{i^{*}}^{\text {down }}$, with "up" or "down" referring to the direction along the magnetic field from which each analyzer collects ions. The ion temperature asymmetry has the same 
qualitative dependence on flow as the asymmetry of ion saturation currents which is exploited by Mach probes, i.e. the effective ion temperature and the ion saturation current are both higher on the side of the probe that collects ions propagating in the direction of the unperturbed bulk flow. With reasonable accuracy, assuming $Z=Z_{f u e l}$, the unperturbed $T_{i}$ is approximately $\left(T_{i^{*}}^{u p}+T_{i^{*}}^{\text {down }}\right) / 2[18]$.

\section{Impurity ion fluxes in the SOL}

In tokamaks, ion species other than fuel ions are labeled as impurities. With the exception of controlled seeding, impurity ions are produced mainly by plasma-wall interactions (e.g. sputtering, arcing, evaporation) or by vacuum imperfections.

For the purpose of our modelling, it would be convenient to know the typical absolute impurity ion fluxes in each charge state striking the RFA. There is, however, little experimental information about the impurity ion fluxes in the SOL. Table 1 compiles some of the available data, indicating that carbon is typically the dominant impurity, followed by oxygen, boron, and metals. Note that in the SOL of ASDEX-Upgrade (AUG) - the divertor tokamak operating from 2007 with a full tungsten wall coating - the relative particle fluxes of metallic impurities are negligible compared to that of residual carbon $\Gamma_{C} / \Gamma_{D}<1 \%$ [19]. Measured relative carbon fluxes are roughly consistent with the erosion/re-deposition pattern (sticking coefficient of carbon ions $\sim 1$, erosion rate of a few percent). As noticed earlier, the impurity ion fluxes measured by PIMS in the SOL of DITE, $\Gamma_{i m p} / \Gamma_{H} \approx 0.5$ [9], represent a rather extreme case [16], and cannot be considered as representative of what might be expected in other tokamaks. The sparseness of the dataset in Tab. 1 does not permit firm conclusions about the difference between the impurity ion fluxes to the limiter and in the main SOL. One could, however, argue that the probe inserted in the SOL acts in a similar way as limiter so that the impurity fluxes to the probe should be comparable to those striking the limiter, i.e. an appreciable fraction of the impurities striking the probe were likely eroded from the probe itself.

\begin{tabular}{|c|c|c|c|c|c|c|c|c|}
\hline \multirow[t]{2}{*}{ Experiment } & \multicolumn{6}{|c|}{ Impurity flux relative to $\mathrm{D}^{+}[\%]$} & \multirow[t]{2}{*}{ Technique } & \multirow[t]{2}{*}{ Location } \\
\hline & $\mathrm{C}$ & $\mathrm{O}$ & B & metals & $\mathrm{Fe}$ & $\mathrm{W}$ & & \\
\hline ASDEX [20] & - & $<0.1$ & - & - & $<0.01$ & - & collector probe & main SOL \\
\hline AUG [19] & $<1$ & $<0.1$ & $<1$ & - & $<0.1$ & - & spectroscopy & pedestal top \\
\hline AUG [21] & - & - & - & - & $<0.5$ & $<0.01$ & collector probe & main SOL \\
\hline C-Mod [10] & - & 1 & $3\left(\mathrm{~B}^{3+}\right)$ & - & - & - & omegatron IMS & main SOL \\
\hline TEXTOR [22] & - & $<0.5$ & - & - & $<0.3$ & - & collector probe & main SOL \\
\hline TEXTOR [23] & - & $<2$ & - & $<0.5$ & - & - & collector probe & main SOL \\
\hline TEXTOR [14] & $12-14$ & 1.3 & 4.4 & - & - & - & PIMS & main SOL \\
\hline TEXTOR [24] & $1-5$ & $1-3$ & $0.1-1$ & - & - & - & spectroscopy & limiter \\
\hline Tore Supra [25] & $1-2.5$ & - & - & - & - & - & particle balance & limiter \\
\hline
\end{tabular}

Table 1. Approximate values of the impurity ion fluxes relative to $D^{+}$measured in the SOL of different tokamaks. Also indicated is the experimental technique as well as the location of the measurements. Because of large uncertainties in the collection efficiencies of $D^{+}$on collector probes [23], the maximum relative impurity flux measured by the collector probes was estimated by dividing the impurity ion particle flux density by $\Gamma_{D} \approx n_{e} c_{s}=10^{22} \mathrm{~m}^{-2} \mathrm{~s}^{-1}$ (with $n_{e}$ being the electron density and $c_{s}=\sqrt{e\left(T_{i}+T_{e}\right) / m_{D}}$ the deuterons sound speed). $\Gamma_{D}=10^{22} \mathrm{~m}^{-2} \mathrm{~s}^{-1}$ is about the minimum estimate of the $D^{+}$particle flux density in the SOL, assuming $n_{e}=10^{18} \mathrm{~m}^{-3}$ and $T_{i}=T_{e}=10 \mathrm{eV}$. 
We conclude from the observations in Tab. 1 that the total impurity ion flux to a RFA is typically dominated by carbon with $\Gamma_{C} / \Gamma_{D}<14 \%$. For the typical range of the SOL electron temperatures $T_{e} \approx 10-50 \mathrm{eV}, \mathrm{C}^{4+}$ will be the principal ionization state [26]. Therefore, in our model, we will consider mainly the mixture of $\mathrm{C}^{4+}$ and $\mathrm{D}^{+}$. Since the thermalization time of $\mathrm{C}^{4+}$ with $\mathrm{D}^{+}$is much shorter than its lifetime, we assume throughout this paper equal temperatures for impurity and fuel ions in the scrape-off layer far from the probe. This is a reasonable assumption also for $\mathrm{C}^{3+}, \mathrm{O}^{4+}$ and $\mathrm{O}^{6+}$, the ion species that will be also considered here as impurities in a mixture with $\mathrm{D}^{+}[9,13,27]$.

\section{Simulation of RFA measurements in an impure plasma}

In order to evaluate RFA measurements in impure plasmas, one clearly needs information about the parallel ion velocity distributions to calculate the corresponding I-V characteristic using Eq.(1). To account for the influence of the pre-sheath electric field on the sheath-edge distribution functions, we generalize the 1D collisionless kinetic model of Chung and Hutchinson [17] to include impurity ions. The equilibrium distribution function $f_{\mathrm{j}}$ of each charge species $j$ is described by a stationary Vlasov equation

$$
\left[\frac{\partial}{\partial z} v+\frac{\partial}{\partial v} \frac{q_{j}}{m_{j}} E(z)\right] f_{j}(v, z)=W\left[f_{j 0}(v)-f_{j}(v, z)\right]
$$

where $z$ is the parallel coordinate, $v$ the parallel speed, $q_{j}$ the charge, $m_{j}$ the mass, and $E$ the parallel electric field. The source term on the right hand side of Eq. (3) represents the exchange of particles between the pre-sheath and the unperturbed, flowing SOL plasma at the characteristic frequency $W$ which we suppose is independent of velocity and of charge species. The unperturbed distribution functions are drifting Maxwellians,

$$
f_{j 0}=n_{j 0} \sqrt{\frac{m_{j}}{2 \pi k T}} \exp \left(-\frac{m_{j}\left(v-U_{d}\right)^{2}}{2 k T}\right)
$$

where $n_{j 0}$ is the particle density of each species far from the probe, and the drift speed $V$ and temperature $T$ are common to all species. Quasineutrality is imposed,

$$
e n_{e}=\sum_{j} q_{j} n_{j}
$$

and the self-consistent electric field is given by the Boltzmann relation for isothermal electrons

$$
E=-\frac{k T_{e}}{e n_{e}} \frac{d n_{e}}{d z}
$$

where $n_{\mathrm{e}}$ is the total electron density. Despite the absence of collisions, the solution of Eq. (3) for each individual species will depend on the concentrations of the other species present because of coupling introduced by the electric field. The calculation begins by imposing an arbitrary electric field profile and solving Eq. (3) for each $f_{j}$ by the method of characteristics. Then the densities of each species are calculated and the electric field is slightly relaxed towards Eq. (6). This process is iterated until the electric field and the density profiles 
converge to a self-consistent solution. For example, the sheath-edge distribution functions $f_{\text {s.e. }}$ of $\mathrm{C}^{4+}$ and $\mathrm{D}^{+}$are illustrated in Fig. 2. In what follows, all velocities are normalized to the cold hydrogen sound speed $c_{H}=\sqrt{e T_{e} / m_{H}}$.
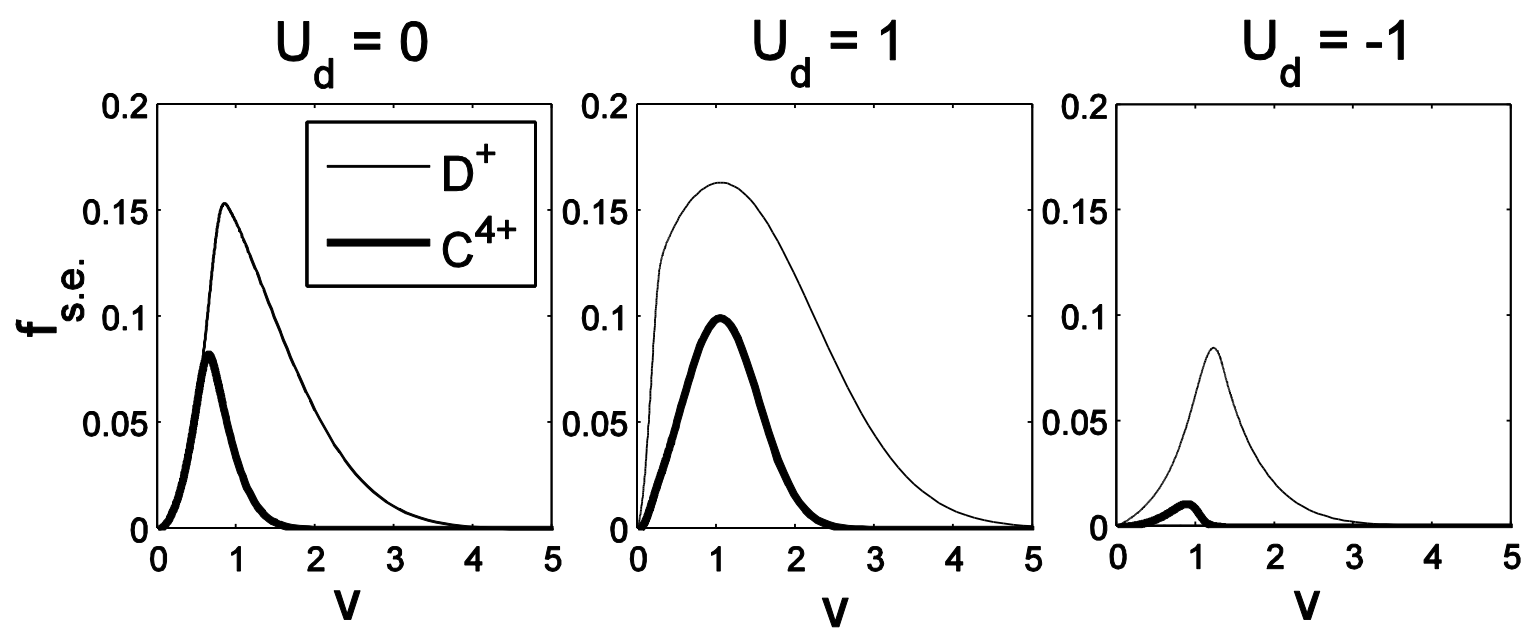

Figure 2. Parallel velocity distributions of $D^{+}$and $C^{4+}$ at the sheath edge obtained from kinetic simulations of the collisionless pre-sheath. Ion-to-electron temperature ratio $\tau=$ 3 and charge density of $C^{4+}$ relative to $D^{+} \quad \rho_{C 4+}=0.5$. Positive (negative) plasma drift velocity $U_{d}$ corresponds to drift towards (away from) the probe. All velocities are normalized to the cold hydrogen sound speed.

The code simulates the collisionless pre-sheath, i.e. the ions proceed to the sheath edge without interspecies collisions. Each ion species thus arrives at the sheath edge with its own sound speed. To compare with experimental measurements (Tab. 1) we need to calculate the relative particle flux at the sheath edge $\Gamma_{\text {imp }} / \Gamma_{\text {fuel }}=\int d v v f_{\text {s.e.imp }} / \int d v v f_{\text {s.e. fuel }}$. Fig. 3 illustrates the relation between $\Gamma_{i m p} / \Gamma_{\text {fuel }}$ and $\rho \equiv Z_{i m p} n_{i m p} / n_{\text {fuel }}$ for selected impurity ion species in a deuterium plasma $\left(Z_{\text {fuel }}=1\right)$. This relation allows identifying the relevant range of $\rho$ to be assumed in the simulations. As seen from Fig. 3, the anticipated range of the relative flux of the principal impurity ion charge state in the SOL $\Gamma_{C 4+} / \Gamma_{D+} \sim<14 \%$ (Sec. 3) corresponds to $\rho_{C 4+} \sim<0.5$. For oxygen, $\left(\Gamma_{O} / \Gamma_{D+} \sim<3 \%\right.$, with the typical ionization states $\mathrm{O}^{4+} \rightarrow \mathrm{O}^{6+}$ for relevant SOL $\left.T_{e} \approx 10-50 \mathrm{eV}[14,26] \mathrm{Tab} .1\right) \rho_{O} \sim<0.1$.

In contrast to the collisionless assumption of the model, in many plasma parameter regimes, different ion species can be collisionally coupled in the pre-sheath, depending on the momentum transfer time relative to the pre-sheath transit time [26]. In the collisional limit, all ions enter the sheath with a common sound speed (given by Eq. (28) in [26] and observed experimentally in [28]) so that $\Gamma_{\text {imp }} / \Gamma_{\text {fuel }}=\rho\left(Z_{\text {fuel }} / Z_{\text {imp }}\right)$. The similar dependence of $\Gamma_{\text {imp }} / \Gamma_{\text {fuel }}$ on $\rho$ in both pre-sheath collisionality limits suggests, Fig. 3, that comparable results would be obtained if interspecies collisions were included in the kinetic model. 


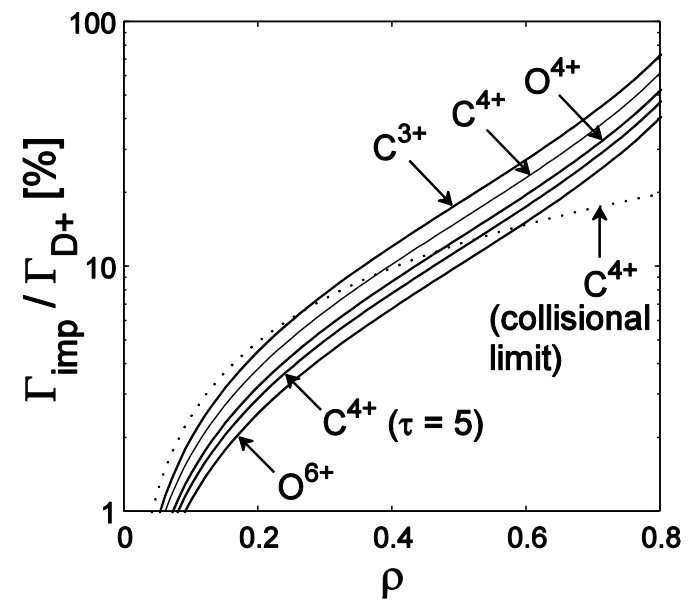

Figure 3. Relative impurity particle flux at the sheath edge plotted against the impurity charge density in the SOL relative to $D^{+}$for relevant impurity ion species (Tab. 1). $\Gamma_{i m p} / \Gamma_{D+}$ has been obtained by integrating the parallel ion velocity distributions calculated from the kinetic simulations assuming the plasma drift velocity $U_{d}=0$, ion-to-electron temperature ratio $\tau=1$, and the collisionless pre-sheath. For $C^{4+}$ (typically the most abundant impurity charge state in the tokamak SOL) the relations between $\Gamma_{i m p} / \Gamma_{D+}$ and $\rho$, obtained for $\tau=5$ as well as for the collisional pre-sheath, are also illustrated for comparison. The collisional limit is obtained from $\Gamma_{\text {imp }} / \Gamma_{D+}=\rho / Z_{\text {imp }}$ (see text).

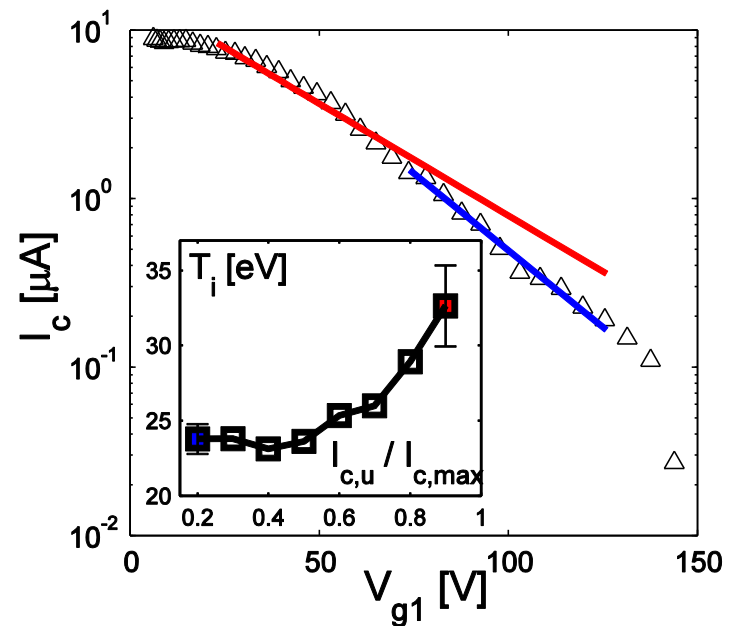

Figure 4. RFA ion I-V characteristic measured in the Tore Supra SOL by the downstream analyzer (ohmic discharge \#35880 in $D^{+}$, distance from the $L C F S r-r_{L C F S} \cong 2 \mathrm{~cm}, U_{d} \cong-0.7$, $\tau \cong 3$ ). The collector current is plotted against the voltage applied to ion repelling grid 1 . The inset panel shows the effective ion temperature obtained from the exponential fit, Eq.(3), to the collector current $-4<\ln \left(I_{c} / I_{c, \max }\right) \leq \ln \left(I_{c, u}\right)$ (where $I_{c, \max }=\max \left(I_{c}\right)$ ) as a function of the upper limit of $I_{c}$ included in the fit, $I_{c, u}$. Also illustrated are the exponential fits for $I_{c, u} / I_{c, \max }=0.2$ and 0.9 . 
The traditional view of probe interactions with magnetized plasmas is that of a long field-aligned pre-sheath, or wake, extending in both directions along the magnetic field. The length of the wake is determined by the balance between particle loss to the probe and the slow diffusive influx of unperturbed plasma from neighboring flux tubes. This model only applies in the absence of cross-field drifts. More generally, in the SOL the radial electric field drives a relatively fast poloidal drift, giving the ions two degrees of freedom rather than only one as in the case of pure parallel flow [29]. As a result, the wake is much more localized around the probe and indeed random walk diffusion can be totally neglected in the formulation of the problem, as shown by collisionless 2D kinetic calculations [30], an elegant 2D fluid analysis employing the method of characteristics [31], and recently confirmed by exact 3D calculations [32]. Therefore, the much shorter transit time of ions across the 2D wake means that the collisionless assumption used in kinetic models has more of a chance to be valid than in the zero drift case, where ion-ion collision mean free paths can be much shorter than the pre-sheath length.

The simulated ion-to-electron temperature ratio $\tau_{f i t}=\left(T_{i^{*}}^{u p}+T_{i^{*}}^{\text {down }}\right) / 2 T_{e}$ is obtained from fitting Eq. (2) to the decaying part of the upstream and downstream ion I-V characteristics constructed from the $f_{j}$ using Eq. (1). The error on $T_{i}$ is thus given by $\tau_{f i t} / \tau$.
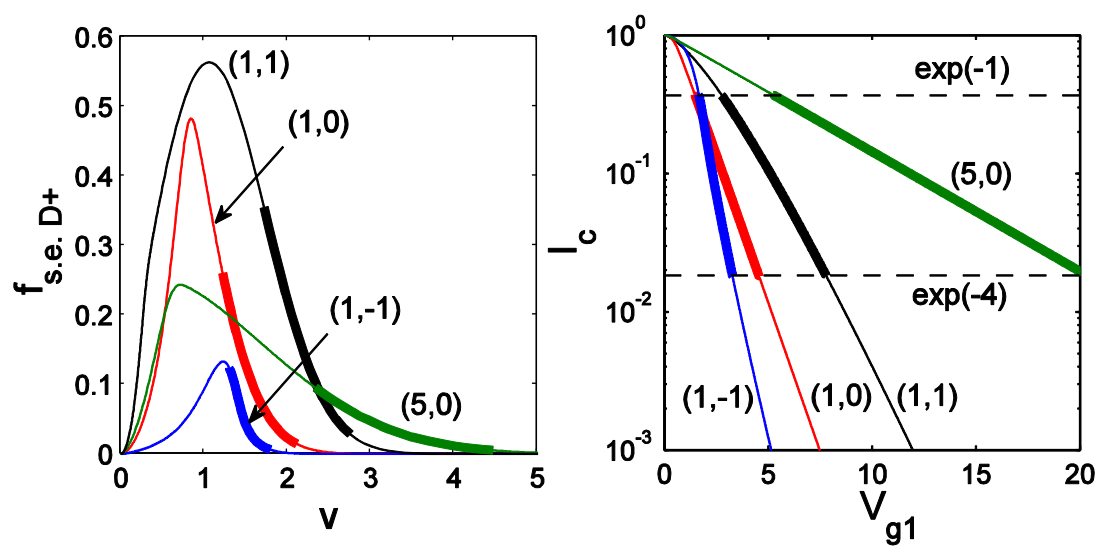

Figure 5. Left: parallel velocity distributions of deuterons at the sheath edge $f(v)_{\text {s.e. }}$ calculated from the kinetic simulations for different ion-to-electron temperature ratios $\tau$ and ion drift velocities $U_{d}\left(\tau, U_{d}\right)$ assuming pure $D^{+}$plasma. $U_{d}$ is positive when directed towards the probe. Right: I-V characteristics obtained from $f(v)_{\text {s.e. }}$ using Eq. (1). Collector current $I_{c}$ is normalized to unity. $V_{g 1}$, normalized to $T_{e}$, equals to $u^{2} A /(2 Z)$. Broad lines indicate the high-energy range of the $I_{c},-4<\ln \left(I_{c}\right) \leq-1$, (and the corresponding portion of $f(v)_{\text {s.e. }}$ ) included in the fit, Eq. (3). The fit gives $\tau_{\text {fit }}=1.03$ (simulations for $\tau=1$ and $U_{d}=0$ ), $\tau_{\text {fit }}=5.03 \quad\left(\tau=5, U_{d}=0\right), \quad \tau_{\text {fit }}^{\text {down }}=0.50 \quad\left(\tau=1, U_{d}=-1\right), \quad \tau_{\text {fit }}^{u p}=1.76 \quad\left(\tau=1, U_{d}=1\right)$ with $\tau_{\text {fit }}=\left(\tau_{\text {fit }}^{u p}+\tau_{\text {fit }}^{\text {down }}\right) / 2=1.13$ [18]. $\tau_{\text {fit }} / \tau>1$ is due to the influence of the pre-sheath (nonexponential) part of the $I-V$ characteristic. 


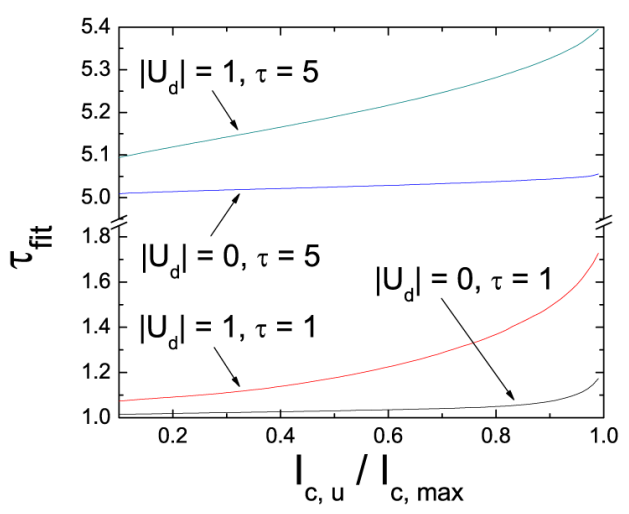

Figure 6. The ion-to-electron temperature ratio $\tau_{\text {fit }}$ obtained from the exponential fit, Eq. (3), to the ion I-V characteristics constructed from the parallel velocity distributions of $D^{+}$at the sheath edge $f(v)_{\text {s.e. }}$ using Eq.(1). $f(v)_{\text {s.e. }}$ were calculated from the kinetic model assuming different input ion-to-electron temperature ratio $\tau$, ion drift velocities $U_{d}$, and pure $D^{+}$ plasma. $\tau_{\text {fit }}$ is plotted as a function of the upper limit of the range of the collector current $I_{c}$ included in the fit, $I_{c, u}$, i.e. $-4<\ln \left(I_{c} / I_{c, \max }\right) \leq \ln \left(I_{c, u}\right)$ where $I_{c, \max }=\max \left(I_{c}\right)$. Vertical dotted line indicates $\ln \left(I_{c, u}\right)=-1$.

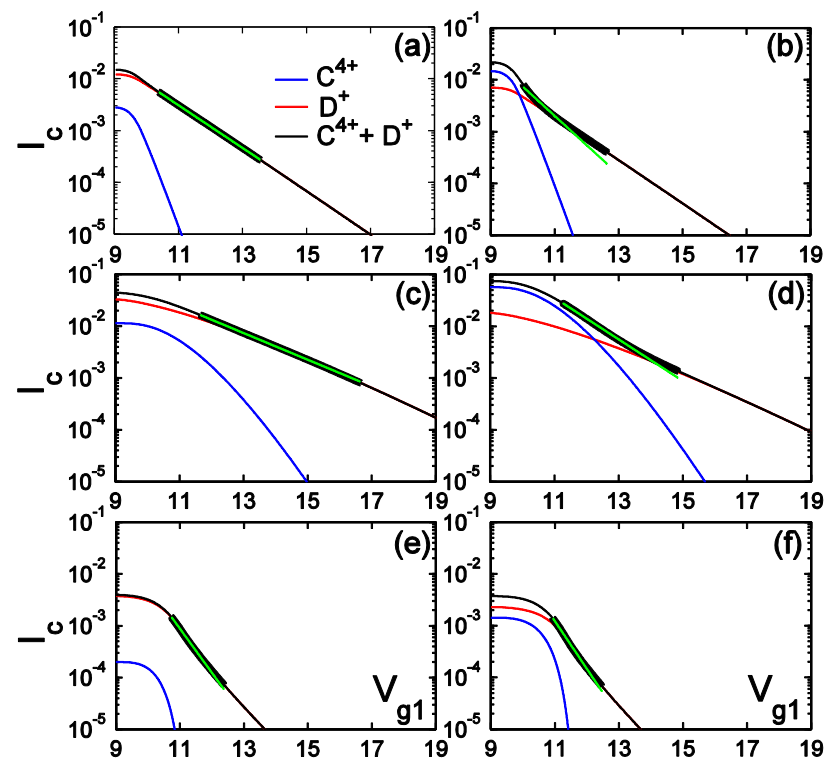

Figure 7. Modelled RFA ion I-V characteristics in the plasma consisting of $D^{+}$and $C^{4+}$ ions, obtained assuming $\tau=1$ and $\varphi / T_{e}=9$ using the procedure outlined in Sec. 4. $V_{g l}$, normalized to $T_{e}$, equals to $u^{2} A /(2 Z)$. Also shown are the individual I-V characteristics of $D^{+}$and $C^{4+}$. Broad lines indicate the high-energy part of the collector current $-4<\ln \left(I_{c} / I_{c, \max }\right) \leq-1$ included in fit (also plotted). $\tau_{f i t}$, the ion-to-electron temperature ratio obtained from the fit, is stated in Tab. 2. $(a, b): U_{d}=0,(c, d): U_{d}=1,(e, f): U_{d}=-1$. Plasma drift velocity $U_{d}$ is normalized to the cold hydrogen sound speed. Positive (negative) $U_{d}$ corresponds to drift towards (away from) the probe. The charge density of $\mathrm{C}^{4+}$ impurity ions in the SOL $\rho_{\mathrm{C} 4+}=0.1$ (first column) and 0.5 (second column). 
In addition to the asymmetry in $T_{i^{*}}$, the kinetic simulations reveal a strong distortion of $f$ in the pre-sheath that has an important consequences for RFA $T_{i}$ measurements, even in the absence of parallel flow. Approaching the probe from the unperturbed plasma, the ion density decreases due to absorption at the probe surface. The depletion of ion density is accompanied by a self-consistent parallel electric field which accelerates ions in the direction of the probe ([17, 18] and Fig. 1 of Ref. [33]). Ions in the high velocity tail of the distribution flow to the probe along phase space characteristics that originate in the unperturbed plasma. The initial probability densities on those characteristics are only slightly modified by the source term in Eq. (3), so the high velocity part of the distribution retains its Maxwellian character. On the other hand, the low velocity range is populated by ions that diffuse into the pre-sheath onto characteristics that originate on the probe with negative velocities, flow a short distance into the pre-sheath, reverse, and return to the probe. The initial probability density is zero at the probe due to the absence of ion emission from the surface, so the low velocity part of the distribution is entirely determined by the source term. Since the typical time of flight to the probe from the point where an ion first appears is insufficient for the local probability density to come to equilibrium, shape of the distribution is not Maxwellian at low $v$. As a consequence, the upper part of the RFA I-V characteristic - the integral of $f$, Eq. (1) is rounded off for $V_{g 1}$ just above $V_{s}$, before the collector current starts to decrease exponentially. A smooth transition between constant $I_{c}$ for $V_{g l}<V_{s}$ and exponentially decreasing $I_{c}$ is often observed experimentally. As shown in Fig. 4, erroneously high $T_{i}$ can be obtained if the pre-sheath (non-exponential) part of the ion I-V characteristic is included in the fit Eq. (2). Figures 5 and 6 show that the influence of the pre-sheath part of the I-V characteristic on $T_{i}$ measurements is stronger for $U_{d} \neq 0$ (with the plasma drift velocity $U_{d}=V / c_{H}$ ) and low $\tau$, for which the pre-sheath part of the I-V characteristic comprises a broader range of $V_{g 1}$. Valsaque et al. [18] proposed to restrict $I_{c}$ included in the fit as $-4 \leq \ln \left(I_{c} / I_{c, \max }\right) \leq-1$, where $I_{c, \max }=\max \left(I_{c}\right)$. As shown in Figs. 5 and 6, such a restriction effectively decreases (though not eliminates) the influence of the pre-sheath part of the I-V characteristic on $T_{i}$ measurements. In what follows, the fit to the I-V characteristic is therefore restricted to $-4 \leq \ln \left(I_{c} / I_{c \text {, max }}\right) \leq-1$, referred to as "high-energy" part of $I_{c}$.

We apply the following procedure to simulate the RFA measurements in an impure plasma:

(i) $\quad f_{\text {s.e. }}$ of fuel and impurity ions are calculated from the collisionless Vlasov code for given $\rho, \tau$, and $U_{d}$. We always assume a single impurity ion species and charge state mixed with $\mathrm{D}^{+}$.

(ii) All ions are accelerated in the Debye sheath potential drop between the sheath entrance and the negatively biased RFA slit plate (Fig. 1) $\varphi=V_{s p}+V_{s}$, i.e. $v^{2} \rightarrow v^{2}+2 Z_{j} \varphi /\left(T_{e} A_{j}\right)$, where $\mathrm{j}$ denotes the impurity or fuel ions and $A$ is the atomic mass.

(iii) The I-V characteristic is obtained from Eq.(1) with $\xi_{r}$ given by Eq. (6) in [12].

(iv) $\tau_{f i t}$ is obtained from the fit Eq. (2) to the high-energy part of the I-V characteristic, assuming $Z=Z_{\text {fuel }}$ as in experiment. 
The simulations are executed for $\left|U_{d}\right|=0 \rightarrow 1$ and $\tau=1 \rightarrow 5$, the range typically measured in the tokamak SOL (e.g. [1, 34]). In order to reduce the number of free parameters, but without loss of generality, $\xi_{r}$ is evaluated assuming the slit dimensions $w=30 \mu \mathrm{m}$ and $s=100 \mu \mathrm{m}$ (Fig. 1). $\xi_{r}$ increases with the increase of $v$, so that the ion transmission through the slit is selective in the parallel direction and leads to a few percent overestimation of $T_{i}$ $[11,12]$. The effect of the slit transmission on $T_{i}$ is, however, weakly sensitive on the slit dimensions (as shown in Fig. 11 in [12], a variation of $s / w$ within a factor of 2 leads to a variation of $T_{i}$ of only a few percent). $\varphi$ is also a free parameter of the model. Typically, $V_{s p}=-100 \mathrm{~V}$ and $V_{s} \approx 3 \mathrm{~T}_{e}$, so that $\varphi / T_{e} \approx 4 \rightarrow 13$ for $T_{e}=10-50 \mathrm{eV} . \varphi$ determines only the ion acceleration in the Debye sheath, which, in turn, affects the ion slit transmission. Since the slit transmission has only a weak effect on $T_{i}$ measurements (for example, for $\tau=1, U_{d}=0$, $\mathrm{D}^{+}$plasma, and $\varphi / T_{e} \approx 4 \rightarrow 13$, the slit transmission leads to overestimations of $T_{i}$ by $9 \% \rightarrow$ $5 \%$, respectively), in what follows we arbitrarily choose $\varphi / T_{e}=9$.

Fig. 7 illustrates the $\mathrm{I}-\mathrm{V}$ characteristics obtained from steps (i)-(iii) with $\mathrm{C}^{4+}$ as impurity ions. The simulations were executed for $\rho_{C 4+}=0.1$ and $0.5, U_{d}=-1,0,1$ and $\tau=1$. The values of $\tau_{\text {fit }}$ are listed in Tab. 2. For $\rho_{C 4+}=0.1$ the contribution of $\mathrm{C}^{4+}$ ions to the total collector current is negligible and $\tau_{f i t}$ is overestimated up to $10 \%$ (which coincides here with the error on $T_{i}$ ) due to the pre-sheath and the ion slit transmission. For $\rho_{C 4+}=0.5$, the I-V characteristics are clearly affected by $\mathrm{C}^{4+}$ at low $V_{g 1}$. However, because of higher $Z$ of $\mathrm{C}^{4+}$, their current to the collector decreases a factor of 4 faster with the increase of $V_{g 1}$ compared to $\mathrm{D}^{+}$ions. The high-energy part of the I-V characteristic is, therefore, only weakly affected by impurity ions even for the maximum anticipated $\rho_{C 4+} \cdot \mathrm{C}^{4+}$ ions lead up to $23 \%$ underestimation of $\tau_{f i t}$, Tab. 2.

\begin{tabular}{lll}
\hline & $\rho_{C 4+}=0.1$ & $\rho_{C 4+}=0.5$ \\
\hline$U_{d}=0$ & 1.05 & 0.77 \\
$U_{d}=1$ (upstream) & 1.69 & 1.08 \\
$U_{d}=-1$ (downstream) & 0.51 & 0.47 \\
$\left(\tau_{\text {fit }}^{u p}+\tau_{\text {fit }}^{\text {down }}\right) / 2$ & 1.10 & 0.77 \\
\hline
\end{tabular}

Table 2. Ion-to-electron temperature ratio $\tau_{\text {fit }}$ obtained from the exponential fit, Eq.(3), to the high-energy part of the RFA I-V characteristics plotted in Fig. 7. $\rho_{C 4+}$ is the SOL charge density of $C^{4+}$ impurity ions relative to $D^{+}$. For $U_{d} \neq 0$, the plasma drift velocity normalized to cold hydrogen sound speed, $\tau_{\text {fit }}$ is calculated separately for each side of the RFA, with upstream/downstream indicating the analyzer facing (aligned with) the plasma flow direction. Also indicated is the average ("unperturbed") $\tau_{f i t}[18]$. 

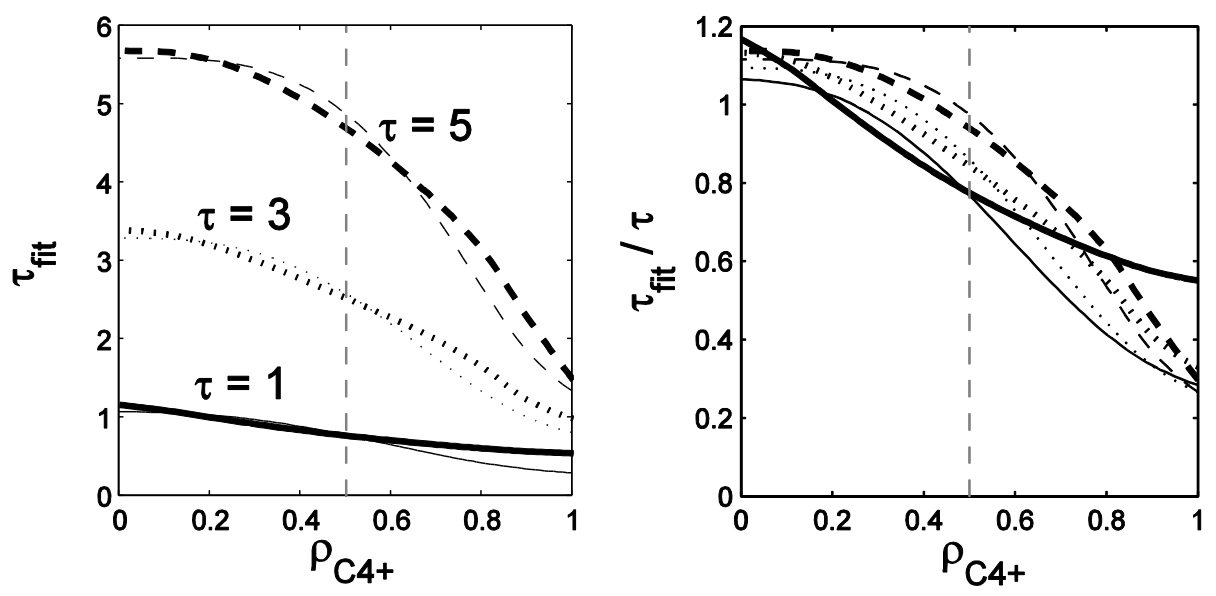

Figure 8. Left: ion-to-electron temperature ratio $\tau_{\text {fit }}$ obtained from the exponential fit Eq.(3) to the modelled RFA I-V characteristics. $\tau_{f i t}$ is plotted against the SOL charge density of $C^{4+}$ relative to $D^{+}$. Right: $\tau_{f i t}$ normalized to the input ion-to-electron temperature ratio $\tau$. Normal lines: $U_{d}=0$, bold lines: $\left|U_{d}\right|=1$, the plasma drift velocity normalized to the cold hydrogen sound speed. Vertical line: anticipated maximum value of $\rho_{\mathrm{C} 4+}$ in the SOL.

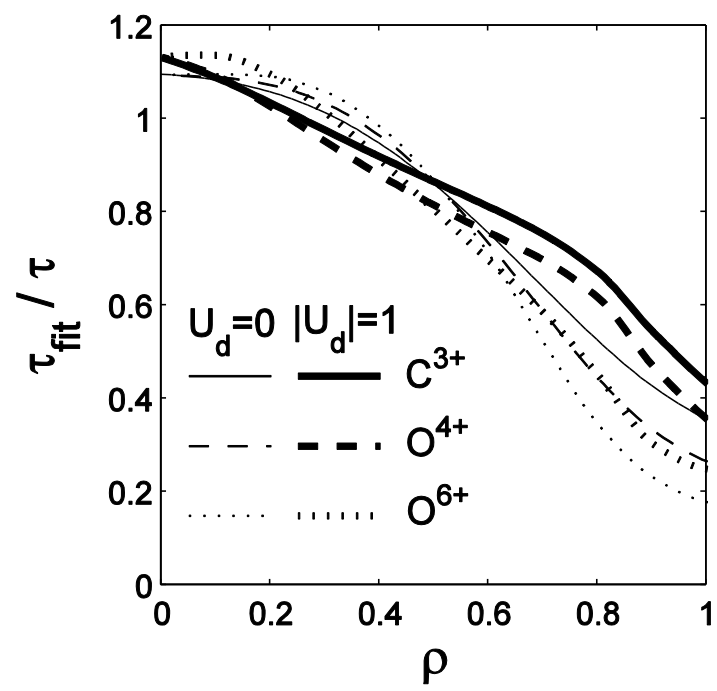

Figure 9. Ion-to-electron temperature ratio $\tau_{f i t}$ obtained from the exponential fit Eq.(3) to the modelled RFA I-V characteristics. $\tau_{f i t}$ is normalized to $\tau=3$ assumed in the simulations and is plotted against the charge density of impurity ions $\mathrm{C}^{3+}, \mathrm{O}^{4+}$ and $\mathrm{O}^{6+}$ relative to $\mathrm{D}^{+} . \mathrm{U}_{d}$ is the plasma drift velocity normalized to the cold hydrogen sound speed.

The influence of $\mathrm{C}^{4+}$ impurity ions on $\tau_{f i t}$ is illustrated for a broader range of $\rho_{C 4+}=0 \rightarrow 1, \tau=1,3$ and 5, and $\left|U_{d}\right|=0$ and 1 in Fig. 8. As demonstrated earlier, for small $\rho_{C 4+}, \tau_{f i t}$ is overestimated due to the pre-sheath effect and the ion transmission through the slit. As $\rho_{C 4+}$ increases, the impurity ions start to affect the high-energy part of the I-V characteristics and $\tau_{f i t}$ becomes underestimated (note that for $\rho_{C 4+}=1, \tau_{f i t}$ is somewhat higher than $\tau / 4$, in contrast to what one would expect from Eq. (3), because the pre-sheath effect and the ion slit transmission tend to overestimate $\tau_{f i t}$ for any $\left.\rho_{C 4+}\right)$. However, for the 
anticipated range of $\rho_{C 4+} \sim<0.5$, the error on $\tau_{f i t}$ remains below $23 \%$. Note that the error on $\tau_{\text {fit }}$ is lower for $\tau>1$ (up to $16 \%$ and $6 \%$ for $\tau=3$ and $\tau=5$, respectively) which is typically observed in the tokamak SOL [1]. Interestingly, for intermediate $\rho_{C 4+}$ within the range anticipated from past measurements, the influence of impurity ions on $\tau_{\text {fit }}$ roughly cancels that of the pre-sheath and the ion slit transmission.

So far, the effect of impurities was investigated assuming only $\mathrm{C}^{4+}$ as impurity ions. Although the model can simulate a mixture of several impurity ion species, the lack of knowledge of the absolute impurity ion fluxes in each charge state introduces considerable freedom in the input parameters. However, as illustrated in Fig. 9, other relevant impurity ions $\left(\mathrm{C}^{3+}, \mathrm{O}^{4+}, \mathrm{O}^{6+}\right.$ ) have very similar effect on $T_{i}$ as $\mathrm{C}^{4+}$ (we recall that the anticipated range of $\rho_{O} \sim<0.1$ ). This result is simply understood as the impurity ions considered here have similar mass and charge. This is also very practical result: although we have investigated the influence of impurity ions by considering always only a single impurity ion species, similar effects on $T_{i}$ would be obtained for a mixture of impurity ions.

\section{Summary}

The RFA is the most frequently used diagnostic for SOL $T_{i}$ measurements in tokamaks. $T_{i}$ is inferred assuming $Z=Z_{\text {fuel }}$, the charge of fuel ions. The impurity ions are considered as a small non-perturbing minority and their influence on $T_{i}$ measurements is neglected. In this paper, we tested the validity of this assumption by simulating measurements of a RFA exposed to a mixture of impurity and fuel ion fluxes. In the simulations we have included the influence of the pre-sheath electric field and the ion transmission through the RFA slit plate. The influence of impurity ions was studied for ranges of impurity ion fluxes, ion-to-electron temperature ratios, and plasma drift velocities typically observed in the tokamak SOL. We considered mainly a mixture of $\mathrm{D}^{+}$and $\mathrm{C}^{4+}$ with equal temperatures.

At low impurity ion concentrations, $T_{i}$ is slightly overestimated due to the pre-sheath effect and the selective ion transmission through the RFA slit, whilst at high impurity concentrations the impurity ions tend to underestimate $T_{i}$. For the range of the parameters considered in our study, the expected error on $T_{i}$ is below $23 \%$, i.e. within the typical accuracy of the measurement. We recommend to extract $T_{i}$ from the "high energy" part of the collector signal, which is less affected by impurity ions and the pre-sheath. A good rule of thumb is to fit only the collector current which is lower than half of the maximum current.

Our results apply to similar probes such as the $\mathbf{E} \times \mathbf{B}$ analyzer [3, 35] or IMS [10-11, 13-14]). At the same time, the influence of impurity ions on other ion sensitive probes could be investigated following the pattern outlined in this study.

\section{Acknowledgements}

We would like to thank R. A. Pitts and K. Krieger for helpful discussions.

\section{References}

[1] M. Kočan et al., J. Nucl. Mater. (2010) doi:10.1016/j.jnucmat.2010.08.036.

[2] M. El Shaer, Ph D Thesis, Scientific and Medical University, Grenoble, 1981.

[3] G. F. Matthews, J. Phys. D: Appl. Phys. 17 (1984) 2243.

[4] M. Kočan, Ph D Thesis, University of Nancy, 2009.

[5] H. Kimura et al., Japan. J. Appl. Phys. 18 (1979) 2275.

[6] A. W. Molvik, Rev. Sci. Instrum. 52 (1981) 704.

[7] A. S. Wan, Ph D Thesis, Massachusetts Institute of Technology, 1986. 
[8] A. S. Wan, T. F. Yang, B. Lipschultz and B. LaBombard, Rev. Sci. Instrum. 57 (1986) 1542.

[9] R. A. Pitts, Ph D Thesis, University of London, 1991.

[10] R. Nachtrieb, B. LaBombard and E. Thomas, Rev. Sci. Instrum. 71 (2000) 4107.

[11] R. Nachtrieb, Ph D Thesis, Massachusetts Institute of Technology, 2000.

[12] M. Kočan et al., Rev. Sci. Instrum. 79 (2008) 073502.

[13] G. F. Matthews, J. M. Pedgley, R. A. Pitts and P. C. Stangeby, J. Nucl. Mater. 176-177 (1990) 1032.

[14] G. F. Matthews et al., J. Nucl. Mater. 196-198 (1992) 253.

[15] I. Nedzelskiy, C. Silva, P. Duarte, and H. Fernandes, Contrib. Plasma Phys. 50 (2010) 866.

[16] R. A. Pitts, private communications, 2011.

[17] K.-S. Chung and I. H. Hutchinson, Phys. Rev. A 38 (1988) 4721.

[18] F. Valsaque, G. Manfredi, J. P. Gunn and E. Gauthier, Phys. Plasmas 9 (2002) 1806.

[19] A. Kallenbach et al., Nucl. Fusion 49 (2009) 045007.

[20] E. Taglauer, B. M. U. Scherzer, P. Varga, R. Behrisch, Chen Cheng Kai and ASDEX team, J. Nucl. Mater. 111-112 (1982) 142.

[21] W. Schustereder, K. Krieger, A. Herrmann, V. Rohde and ASDEX Upgrade Team, J. Nucl. Mater. 363-365 (2007) 242.

[22] R. A. Zuhr and W. R. Wampler, J. Vac. Sci. Technol. A 3 (3) (1985) 1188.

[23] M. Rubel, H. Bergsåker, B. Emmoth, P. Wienhold and F. Waelbroeck, Physica Scripta 43 (1991) 508.

[24] J. D. Hey, Y. T. Lie, D. Rusbüldt and E. Hintz, Contrib. Plasma Phys. 34 (1994) 725.

[25] E. Dufour, private communications, 2010.

[26] P. C. Stangeby, J. Phys. D: Appl. Phys. 20 (1987) 1472.

[27] P. Bogen et al., J. Nucl. Mater. 220-222 (1995) 472.

[28] D. Lee, N. Hershkowitz and G. D. Severn, Applied Physics Letters 91 (2007) 041505.

[29] J. P. Gunn, J. -Y. Pascal, F. Saint-Laurent, C. Gil, Contrib. Plasma Phys. 51 (2011) 256.

[30] J. P. Gunn, Czech. J. Phys. 48 (1998) 293.

[31] I. H. Hutchinson, Phys. Rev. Lett. 101 (2008) 035004.

[32] L. Patacchini and I. H. Hutchinson, Plasma Phys. Control. Fusion 52 (2010) 045005.

[33] J. P. Gunn and V. Fuchs, Phys. Plasmas 14 (2007) 032501.

[34] N. Asakura, J. Nucl. Mater. 363-365 (2007) 41.

[35] P. Staib, J. Nucl. Mater. 111-112 (1982) 109. 